\title{
Research on the impact of coal miners' workload on unsafe behaviors
}

\author{
Taozhu Feng ${ }^{1, a}$, Yingsha Ren ${ }^{2, b^{*}}$ \\ ${ }^{1}$ School of Management, Xi'an University of Science and Technology, Xi'an, Shaanxi, China \\ ${ }^{2}$ School of Management, Xi'an University of Science and Technology, Xi'an, Shaanxi, China
}

\begin{abstract}
Workload is one of the reasons that affect unsafe behavior in coal mines. This article divides workload into six dimensions: physical demand, mental demand, time demand, performance level, effort level and frustration level, and introduces miner emotions as an intermediary variable to study the impact of workload on unsafe behavior. Through a questionnaire survey of coal mine workers, a structural equation model of coal mine workers' workload and unsafe behavior is constructed. The results show that the six dimensions of workload have a significant impact on emotions. Physical demand, mental demand, time demand and frustration level have a significant impact on unsafe behaviors. Performance level and effort level have no significant impact on unsafe behaviors.
\end{abstract}

\section{Introduction}

The direct cause of safety accidents is the unsafe behavior of people and the unsafe state of things. According to statistics from experts and scholars, more than $96 \%$ of coal mine accidents are caused by human factors. In recent years, the number of accidents and deaths in coal mining enterprises has decreased year by year, but the safety situation in coal mines still cannot be ignored.

The working environment and nature of the coal industry make it a veritable high-risk industry. The work of coal miners is a rotating operation mode, which takes a long time to work underground. There are a series of preparations before going down and the actual working time of coal miners. Far more than the eight-hour work system. The poor underground environment, heavy tools and equipment, and long hours of daily labor make the coal miners' workload too heavy. Without sufficient rest time, it is easy to cause physical exhaustion, which makes the miners' satisfaction and mental emotions poor, leading to safety status. Numbness can easily lead to unsafe behaviors. Therefore, how to effectively alleviate the work load of coal miners is very important to prevent unsafe behaviors of coal miners and reduce the occurrence of unsafe accidents.

At present, most scholars' research on workload is mostly concentrated in occupational fields such as nurses, teachers, pilots, and construction workers. There are few researches on the workload of coal miners[1-2]; and the research on unsafe behaviors of coal miners is currently Most of them focus on the influence of factors such as safety perception, work pressure, job satisfaction, and subjective well-being on unsafe behavior [3-5]. Few

aemail: 2657620339@qq.com

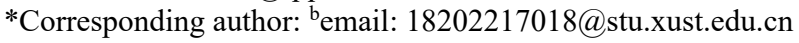

scholars have studied the relationship between coal miners' workload, emotions and unsafe behavior. Therefore, from the perspective of coal miners' workload, this article uses emotion as an intermediary variable to construct a structural equation model of workload-emotion-unsafe behavior, explores the impact of workload on unsafe behavior, and proposes relevant countermeasures.

\section{Theory construction and research hypothesis}

\subsection{Work load and emotions, unsafe behavior}

Workload is one of the variables that have received much attention in the field of organization and management. We call the workload that the human body bears per unit time as workload, which includes both physiological and psychological load. The NASA-TLX workload evaluation system adopted by NASA divides the workload into six aspects: physical demand, mental demand, time demand, performance level, effort level and frustration level. If a person feels powerless or depressed about their work, or their work performance is reduced, or their job satisfaction is too low, the emergence of work overload should be considered.

(1) Physical demand. It is defined as the amount of physical activity that needs to be devoted to work, such as pushing, pulling, carrying, etc. Whether the job is physically easy or strenuous. At present, most of the coal mines in our country are underground mines, which require underground mining. The working space of coal miners is narrow and the machinery and equipment are heavy. Coal miners need to perform a lot of manual labor 
every day to complete their work tasks, which can easily cause physical overload and increase workers' workload. The degree of fatigue, causing unsafe behavior. The daily physical overload of workers can also cause coal miners to feel depressed. Li,M.[6]studied the impact of coal miners' physical load on the risk of industrial accidents, and believed that coal miners' physical overload would increase the probability of errors. Therefore, it can be inferred that physical demand may affect emotions and unsafe behaviors. Based on this, the following hypotheses are proposed: H1a: physical demand has a positive influence on unsafe behavior; H1b: physical demand has a negative influence on emotions.

(2) mental demand. It is defined as how much mental activity needs to be devoted to work, such as memory, thinking, judgment, etc. Whether the work is easy or laborious in mental work, and whether the mind is continuously highly concentrated during work. Miners need not only physical labor, but also mental labor such as memory and judgment. They need to maintain a high concentration of attention at all times during work. Coupled with the influence of underground noise and vision on workers' brains, it is easy to cause miners' brains. Load affects workers' emotions and behaviors. Based on this, the following hypotheses are proposed: $\mathrm{H} 2 \mathrm{a}$ : Mental demand has a positive impact on unsafe behaviors; H2b: Mental demand has a negative impact on emotions.

(3) time demand. It is defined as whether there is a break during work, whether it is a long-term continuous work or a leisurely rest. Is there a normal holiday break for this job? The working hours of coal miners basically adopt a rotating shift system, working eight hours per shift, but this eight-hour work system is only their working time in the underground, plus a series of preparations before going down the well and cleaning after going up. The working hours have been far more than 8 hours. Such long hours of work will cause physical and psychological fatigue, affect workers' emotions, and easily lead to human errors. Therefore, it is inferred that time demand will affect workers' emotions and behaviors, and the following hypotheses are proposed: H3a: time demand has a positive effect on unsafe behavior; $\mathrm{H} 3 \mathrm{~b}$ : time demand has a negative effect on emotions.

(4) Performance level. It is defined as the ability to complete work tasks on time, and whether you are satisfied with your performance after the work is completed. Performance appraisal is the company's evaluation of employees' work behavior and work performance. The level of coal miners' performance is closely related to their salary. Miners with too low levels of performance will also be punished accordingly, which can easily cause negative emotions of miners and affect their behavior. Based on this, it is inferred that performance level will have a certain impact on emotion and behavior, and the following hypotheses are proposed: H4a: performance level has a negative impact on unsafe behavior; $\mathrm{H} 4 \mathrm{~b}$ : performance level has a positive impact on emotions.

(5) Effort level. It is defined whether this work requires you to put in a lot of effort to achieve the established performance and work requirements, whether it is still difficult to complete the task after putting in the effort. Hyde's naive attribution theory believes that the cause of behavior is the environment or the individual, and personal reasons include effort. At present, related scholars believe that the degree of work effort will affect individual behavior. In the coal industry, the complexity of the operation of the coal mine itself, the heaviness of the workload and the intensity of the work, etc., may cause coal miners to make great efforts every day to complete, or make great efforts but still fail to meet the requirements. Workers are prone to passive sabotage, resulting in unsafe behavior. Therefore, the following hypotheses are proposed: H5a: Effort level has a positive effect on unsafe behavior; H5b: Effort level has a negative effect on emotions.

(6) Frustration level. It is defined as whether you feel insecure, frustrated, annoyed, stressed, etc. at work. The frustration theory put forward by Adams believes that from a negative perspective, frustration can make people feel disappointed, frustrated and even depressed. Frustration can also lead to irrational behaviors and cause bad consequences. Therefore, the following hypotheses are proposed: H6a: the level of frustration has a positive effect on unsafe behavior; H6b: the level of frustration has a negative effect on emotions.

\subsection{Emotions and unsafe behaviors}

The emotional event theory mainly explores the relationship between the emotional events and emotional reactions experienced by the members of the organization and their attitudes and behaviors. The theory believes that the characteristics of the work environment will cause the occurrence of positive or negative work events, which will trigger individual emotional reactions and further affect individual attitudes and behaviors. Ma,Y.J.[7] analyzed the needs and emotions of miners in coal mine safety based on the hierarchy of needs theory. She believes that the emotional problems of miners are an important psychological factor affecting unsafe behavior. Therefore, the research hypothesis is proposed: H7: Emotion has a negative influence on unsafe behavior of coal miners. The relational model is shown in Figure 1 . 


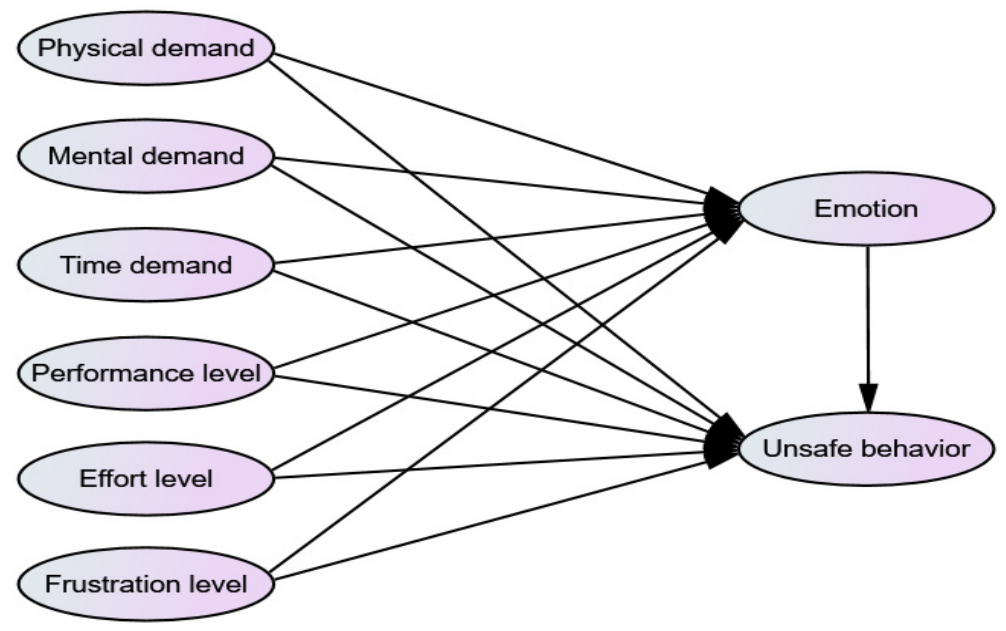

Fig. 1 Conceptual model of workload impact on unsafe behavior

\section{3 research methods}

\subsection{Research objects}

Using a multi-stage sampling method, multiple companies were selected from coal mining companies in Shaanxi and Shanxi, and questionnaires were issued to front-line coal mine employees. A total of 168 questionnaires were issued for the first pre-investigation, and invalid questionnaires such as the same answer to each question were eliminated and recovered. There were 158 valid questionnaires, with an effective response rate of $94 \%$; a total of 250 questionnaires were distributed in the formal survey, and invalid questionnaires were eliminated, and 218 valid questionnaires were recovered, with an effective rate of $87.2 \%$.

\subsection{Research tools}

\subsubsection{Workload Scale}

The six dimensions in NASA-TLX are used as the dimension division to study the workload of coal miners. The items under each dimension are combined with the characteristics of the workload of coal miners and the nature of the industry to form an initial questionnaire. The scale uses Likert's five-point score method (1 means "strongly disagree", 5 means "strongly agree"), in which physical demand include 4 items, mental demand include 3 items, time demand include 3 items, performance level include 3 items, effort level includes 3 items, and frustration level includes 3 items, for a total of 19 items. After the reliability and validity analysis of the initial questionnaire, one question with low validity under the physical load dimension was deleted, and a formal workload scale was formed, with a total of 18 items.

\subsubsection{Emotional Scale}

On the basis of combing the literature, referring to the
Emotion Questionnaire of Mainland scholar Jie Li and Lifang Dai's Negative Emotion Sources Scale, the Emotion Measurement Scale was revised and compiled, which contained one dimension and 6 items. After the pre-investigation, the 2 items with low validity were deleted, and the final formal scale contained 4 items.

\subsubsection{Unsafe Behavior Scale}

At present, the academic circle has relatively mature research on the measurement scale of unsafe behavior. The research object of this article is coal miners. Therefore, the unsafe behavior scale developed by Jianjin $\mathrm{Wu}$ is used to measure the unsafe behavior of coal miners. The scale has one dimension. Contains a total of 8 items. After the pre-investigation, all items meet the reliability and validity requirements, so all items are retained to form a formal unsafe behavior scale.

\section{Empirical analysis}

\subsection{Reliability and validity analysis of the scale}

Use SPSS21.0 software to analyze the validity and reliability of the questionnaire data. Through the analysis, the Cronbach's $\alpha$ values of workload, emotion, and unsafe behavior are $0.840,0.883$, and 0.936 respectively. From the above data, we can see that the reliability of each variable The values are all greater than 0.7 , and the reliability meets the requirements. The overall reliability coefficient is 0.833 , which is high in reliability and within an acceptable range, which shows that the data in the questionnaire is credible. The Bartlett sphere test of variable workload, emotion and unsafe behavior is all less than 0.05 , and the KMO value is all greater than 0.8 , and the validity is appropriate, which shows that the validity of the questionnaire data is guaranteed. The Cronbach's $\alpha$ value and KMO specific data of each variable are shown in Table 1. 
Table 1 Cronbach's $\alpha$ value and KMO value of each scale

\begin{tabular}{ccccc}
\hline Variable & Workload & Mood & $\begin{array}{c}\text { Unsafe } \\
\text { behavior }\end{array}$ & $\begin{array}{c}\text { Total } \\
\text { scale }\end{array}$ \\
\hline Cronbach's $\alpha$ & 0.840 & 0.883 & 0.936 & 0.833 \\
KMO & 0.872 & 0.829 & 0.851 & 0.918 \\
$\begin{array}{c}\text { Bartlett's } \\
\text { sphere test }\end{array}$ & 0.000 & 0.000 & 0.000 & 0.000 \\
\hline
\end{tabular}

\subsection{Model verification and analysis}

\subsubsection{Overall model fit index}

In terms of overall model fit, several more important indexes are usually examined in the fit index. The AMOS 23.0 software was used to test the model, where the $\mathrm{X} 2 / \mathrm{df}$ value was 2.022 , which was within the interval of 1 to 3 , indicating a good fit of the model; the RMSEA value was 0.069 , less than 0.08 , indicating a good fit; NFI, GFI and CFI are 0.852, 0.821 and 0.918, respectively, which are both greater than 0.8 . Combining the above data analysis, we can see that the model has a relatively good fit.

\subsubsection{Analysis of model running results}

The path analysis results of the model are shown in Table 2 , and the path diagram of the impact of workload on the unsafe behavior of coal miners is shown in Figure 2.

Table 2 Hypothesis test results

\begin{tabular}{ccccc}
\hline Assumption & Action pathway & Estimate & $\mathrm{P}$ & Verification \\
\hline $\mathrm{H} 1 \mathrm{~b}$ & Emotion<---Physical demand & -.257 & $* * *$ & valid \\
$\mathrm{H} 2 \mathrm{~b}$ & Emotion<---Mental demand & -.178 & $* *$ & valid \\
$\mathrm{H} 3 \mathrm{~b}$ & Emotion<---Time demand & -.133 & $*$ & valid \\
$\mathrm{H} 4 \mathrm{~b}$ & Emotion<---Performance level & .167 & $* *$ & valid \\
$\mathrm{H} 5 \mathrm{~b}$ & Emotion<---Effort level & -.142 & $*$ & valid \\
$\mathrm{H} 6 \mathrm{~b}$ & Emotion<----Frustration Level & -.281 & $* * *$ & valid \\
$\mathrm{H} 1 \mathrm{a}$ & Unsafe behavior<---Physical demand & .244 & $* *$ & valid \\
$\mathrm{H} 2 \mathrm{a}$ & Unsafe behavior<---Mental demand & .139 & $*$ & valid \\
$\mathrm{H} 3 \mathrm{a}$ & Unsafe behavior<---Time demand & .133 & $*$ & valid \\
$\mathrm{H} 4 \mathrm{a}$ & Unsafe behavior<---Performance level & --- & .710 & invalid \\
$\mathrm{H} 5 \mathrm{a}$ & Unsafe behavior<---Effort level & --- & .863 & invalid \\
$\mathrm{H} 6 \mathrm{a}$ & Unsafe behavior $<---$ Frustration Level & .217 & $* *$ & valid \\
$\mathrm{H} 7$ & Unsafe behavior $<---$ Emotion & -.268 & $*$ & valid \\
\hline
\end{tabular}

P.S.: ${ }^{* * *}: \mathrm{p}<0.001,{ }^{* *}: \mathrm{p}<0.01,{ }^{*}: \mathrm{p}<0.05$.

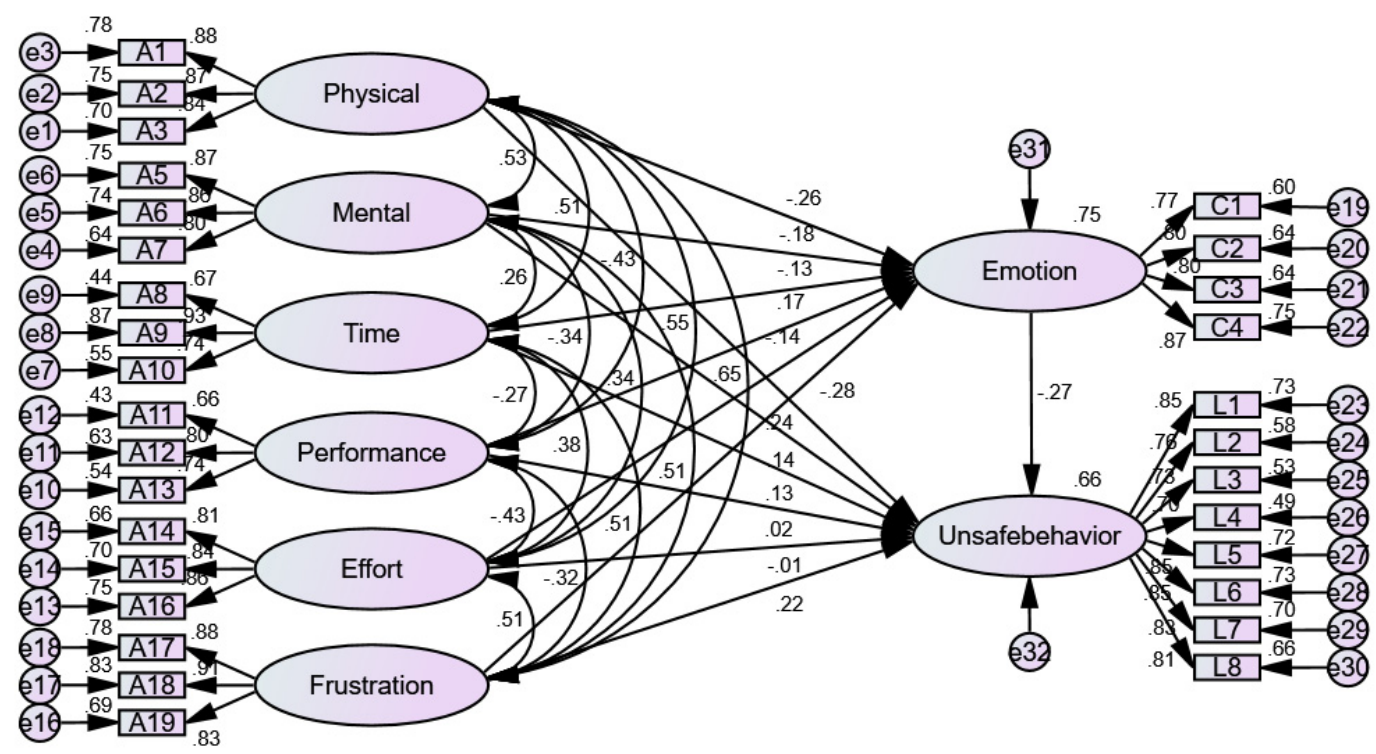

Fig. 2 Influence path diagram of work load on unsafe behavior of coal miners

From the above test results, it is found that the path coefficient of coal miners' emotions and unsafe behaviors is -0.268 , and the $\mathrm{P}$ value is less than 0.05 , indicating that miners' emotions have a negative influence on unsafe behaviors. When coal miners have positive and good emotional responses, will reduce the possibility of unsafe behavior. In the six dimensions of coal miner's workload, the path coefficient between physical demand and emotion is -0.257 , the $P$ value is less than 0.001 , the path coefficient with unsafe behavior is 0.244 , and the $P$ value 
is less than 0.01, indicating the physical demand and unsafe behavior. The impact effect is significant, and the unsafe behaviors of miners are affected through two paths, reducing the physical demand of miners during work, and reducing physical load can avoid unsafe behaviors. The path coefficient of mental demand and emotion is -0.178 , the $P$ value is less than 0.01 , and the path coefficient of unsafe behavior is 0.139 , and the $\mathrm{P}$ value is less than 0.05 , indicating that the mental demand of miners has a significant impact on unsafe behaviors, and reducing the mental load of miners can be reduce the possibility of unsafe behavior. The path coefficient of time demand and emotion is -0.133 , and the path coefficient of unsafe behavior is 0.133 , and the $P$ value is less than 0.05 , indicating that the miner's time demand has a significant impact on unsafe behavior, and reducing the working time of miners can effectively reduce probability of unsafe behavior. The path coefficient of performance level and emotion is $0.167, \mathrm{P}$ value is less than 0.01 , and the $\mathrm{P}$ value of unsafe behavior is equal to 0.710 , indicating that performance level has a significant impact on emotions, and there is no significant impact on unsafe behavior. Therefore, the performance level of miners is passed Influencing the mood of miners indirectly affects unsafe behaviors, and has no direct impact on the occurrence of unsafe behaviors. The path coefficient of effort and emotion is -0.142 , $P$ value is less than 0.05 , and the $\mathrm{P}$ value of unsafe behavior is equal to 0.863 , indicating that the impact of effort and emotion of coal miners is more significant, and there is no significant impact on unsafe behavior.The path coefficient of frustration level and emotion is $-0.281, \mathrm{P}$ value is less than 0.001 , and the path coefficient of unsafe behavior is $0.217, \mathrm{P}$ value is less than 0.01 , indicating that the frustration level affects unsafe behavior through two paths and reduces coal miners' work Frustration can effectively improve the mood of miners and reduce the incidence of unsafe behaviors.

\section{5 recommended measures}

(1) Pay attention to reducing the burden of coal miners The six dimensions of workload have a significant impact on the mood and unsafe behavior of miners. Only by reducing the workload of coal miners can the occurrence of unsafe behaviors be effectively reduced. Therefore, the following measures are proposed:

(1) Speed up coal mine mechanization transformation. Coal mining enterprises must accelerate the speed of mechanized transformation and replace human labor with machines. Through intelligent transformation, not only can they complete production tasks more efficiently and improve the underground environment, but also reduce the number of people going down in a single shift, effectively reducing the labor intensity of coal workers. Reduce the physical load of coal miners.

(2) Cancel night work. The night shift is the most fatigued time period, and it is also the time period when safety accidents are prone to occur. Affected by the biological clock, underground night shift operations cannot achieve a high degree of concentration. Coal miners are prone to wandering during night shift operations, which will increase accidents. Therefore, the cancellation of the three-shift work mode allows coal miners to get adequate rest, which not only reduces safety risks and labor intensity, but also protects the health of coal miners. In addition, coal mine safety supervision and inspection departments should also strengthen supervision and inspection, so that the cancellation of the night shift system can be implemented.

(3) Raise the wages of frontline workers. Enterprises must implement the increase in wages and benefits, make coal miners' efforts to get due rewards, match wages and labor intensity, and increase wage income to the bottom line and people in difficult jobs. The effective increase in wages can increase workers' enthusiasm and enthusiasm for work, keep them in a good mood, and reduce the burden on workers in life, so they can devote themselves to work. Good working attitude and mood can effectively reduce the occurrence of unsafe behaviors of workers.

(4) Reduce the frustration of workers. The leadership department should convey the correct values to coal miners and allow them to establish a correct view of frustration. After frustration occurs, they must consciously carry out psychological defenses and control their emotions and behaviors. Strengthen corporate leadership, subtly influence and strengthen the psychology of coal miners.

(2) Pay attention to the emotional problems of coal miners

(1) Organize entertainment activities. Teams can regularly carry out quality development activities for coal mine workers and hold various entertainment team building activities, which can not only achieve a relaxing effect and keep workers positive and optimistic, but also promote good relationships between colleagues and between workers and leaders.

(2) Pay attention to emotional observation. Coal mining companies can set up emotional management positions according to their own conditions, monitor the emotional changes of coal miners through a variety of methods, and grasp the emotional state of miners in a timely manner. For employees with emotional problems, they are prohibited from going down the well, and emotionally directed, and resolved in communication Emotional problems.

\section{Conclusion}

On the basis of reading relevant literature, combined with the nature and characteristics of my country's coal industry, the dimensions of workload are divided, and corresponding hypotheses are proposed. By constructing a structural equation model for verification and analysis, the final model of workload and unsafe behavior is obtained. The results show that the model fits well. For coal companies, it is possible to improve workers' mood by reducing the workload of coal workers and prevent and control the occurrence of unsafe behaviors. 


\section{References}

1. Tao,M.L.,Qi,S.J.,Cheng,J.L.,Zhang,Y.B.(2019)Study on Structure Relationship between Work Load and Unsafe Behavior of Construction Workers.J. Construction economy. 40:30-35.

2. Xiao,L.S.(2017)Independent college teachers' workload and turnover intention.J.Higher Education Exploration.80-89.

3. Li,H.X.,Li,S.Q.(2017)Research on the Relationship between Coal Miners' Safety Cognition and Unsafe Behaviors.J.coal mine safety.48:233-236.
4. Li,N.W.,Liu,M.X.,Niu,L.X.(2018)The relationship between work pressure, mental wandering and unsafe behavior of miners.J.China Safety Production Science and Technology.14:170-174.

5. Li,J.Z.,Wang,X.J.(2017)Research on Miners' Subjective Well-being and Unsafe Behavior.J.China Coal.43:125-129.

6. Li,M.(2013)Correlation analysis of physical energy load and industrial accidents of Chinese miners.J.Coal technology.32:267-268.

7. Ma,Y.J.(2007)Research on Miners' Mood and Safety. J. China Coal.60-62. 